\title{
Automatic Video System for Aircraft Identification
}

\author{
J.M. Molina, J. García \\ Dpto. Informática \\ Universidad Carlos III de Madrid. \\ Leganés, Madrid, SPAIN \\ molina@ia.uc3m.es \\ jgarcia@inf.uc3m.es
}

\author{
A. Berlanga, J. Besada, J.Portillo \\ GPSS-DSSR- ETSIT \\ Universidad Politécnica de Madrid \\ Madrid, SPAIN \\ aberlanga@grpss.ssr.upm.es \\ besada@grpss.ssr.upm.es \\ portillo@grpss.ssr.upm.es
}

\begin{abstract}
Advanced Surface Movement Guidance and Control Systems need the identification of aircraft and vehicles in airport movement area. In this work, a video identification algorithm, based on the tail number recognition, is proposed as a part of a global surveillance video system. The aircraft identification problem has to deal with three fundamental aspects: capturing and preprocessing the images, the international regulation defining the tail number grammar and the pattern recognition methodology. Considering these aspects, the developed system is based basically on three ideas: in the first place, to use the local grey level contrast to detect the characters of the tail number; in second place, to find the zone where the tail number is written to isolate it of the rest of the image; and finally to process this zone to identify the aircraft, using an aircraft database.
\end{abstract}

Keywords: ASMGCS, Video Surveillance, Aircraft Identification, Image Processing, Pattern Recognition.

\section{Introduction}

Advanced Surface Movement Guidance and Control Systems (A-SMGCS) [1][2][3] requires the unambiguous identification of all aircraft and vehicles in the airport movement area. The sensors used currently (or in the near future) to perform airport surveillance are Surface Movement Radar (SMR)[4], Multilateration systems (MS)[5][6], differential GPS broadcasted through a digital data-link (DGPS) and, finally, Video cameras (as described in this paper).

The developed identification system is part of on-going VICTOR ("Visualización Integrada para Control de TORre" - Integrated Visualisation for Tower Control) project, launched by AENA (Spanish Air Navigation Services Provider) in 1997 as the first step towards the implementation of an A-SMGCS for Madrid/Barajas Airport. Nevertheless, one of the main requisites in this design is that it should be easily deployed in other airports (AENA currently manages all Spanish airports).
Video cameras are a non-cooperative sensor, able to provide both identification and tracking avoiding the need of installing additional avionics on board [7]. The method used to obtain target identification for aircraft is performing an image based tail number recognition, through an optical character recognition (OCR) algorithm. The only restriction of TV cameras is the necessity of clear meteorological conditions that means not too dense fog, rain or snow. Occlusions and operation on bad weather conditions are the main problems of this system.

The identification system described here is part of a global system that provides controllers (and potentially pilots) with a display of the location of all surface traffic [8], enabling its separation and guidance in all types of weather conditions without reducing the number of operations or the level of safety. The whole image-based surveillance system will be designed to perform accurate detection and position measurements of aircraft and vehicles on all airport areas and 2D multitarget tracking [9]. The implemented tracking system [10] is based on a distributed tracking architecture, in which every camera has a dedicated positioning/tracking system which calculates target trajectories (local tracks) in the camera projected plane.

The approach used to obtain aircraft identification has been to use an OCR to recognize aircraft tail number [11]. Cameras for this function should be deployed near the taxiways and runways, in positions being traversed by all the interest targets, prior to their entrance in the area to be controlled (mainly runways and taxiways). When an aircraft passes in front of the camera (which may be predicted using the tracking system described before), an image of its tail is captured. Then, the aircraft identification algorithm is applied.

The problem of aircraft tail number identification in an airport, by means of tail number recognition, comprises three fundamental aspects: how to capture and pre-process the images to identify the tail number, how to use the international regulation that defines the grammar to 
generate tail numbers and which, from among the different character recognition methods in the literature [11][13][14][15], is the most adequate technique to identify each one of the characters than compose the tail number.

The identification algorithm has to search in the image for the presence of the tail number, detecting it in a non necessarily uniform background which besides contains discontinuities, additional letters (aircraft manufacturer, for instance), etc. The method looks for several contrasting regions (the letters), aligned in the figure, and then use an OCR over the local region containing it. The OCR algorithms have been designed to deal with the real images we are going to have, taking into account letter sizes differences, potential inclination, and the ICAO tail number normative [12]. The algorithm then uses the OCR results and a database containing the tail numbers of all aircraft in the airport (or about to land) to identify the aircraft among the potential candidates.

The OCR problem can be solved using different approaches [16] [17] [18], which may be grouped into the following families:

- Approximations based on the correlation of the original image with patterns. In this case, each pattern represents the ideal image of a character. Correlation techniques are the most widely used in OCR, since they provide the best results.

- Feature-based approximations: the problem is converted into a vector classification problem. A set of predefined features are a priori selected to represent each ideal character (pattern). This features are extracted from the real image, and a classifier decides the most appropriate pattern.

- Approximations based on morphological (structural) properties: the pattern are defined by a set of deformable structural properties to be matched with the real image. They are specially used for recognition when there are no predefined patterns, as in the hand written texts.

Spatial transformations, deformations, drafts, etc represent a great problem both for correlation-based and feature-based methods. In the case of the correlation procedures these deformations could be treated previously applying a transformation model either to the patterns or to the captured image. In the case of feature-based method, the image can be treated previously (as in the case of correlation procedure) or a transformation function in the features vector may be used.

The feature-based procedures are more insensitive to problems derived from the variation in the characters aspect (variation from the height, width, letter type, etc.) if this definition is insensitive to such variations. Therefore, we will use a feature-based method defined taking into account these problems. The features we are using are the proportion of the pixels belonging to character, measured in nine equal-size subimages defined by a 3x3 grid uniformly spaced both in horizontal and vertical directions of character region (see Figure 8). This representation is quite insensitive to character aspect variations, while allowing enough disparity among patterns for our application.

Finally, the classification problem can be approached using several techniques, such as test of hypothesis, neural nets, etc. But we do not use a classifier for each character within the tail number. Instead, our approach maximizes the joint probability of the complete tail number for each of the aircraft in the airport database, using the method to be described in section 4 .

In section 2 an analysis of real images from Madrid/Barajas Airport is presented. Section 3 describes identification procedure. Section 4 is devoted to distance calculation among characters and, section 5 is related to database aided aircraft identification. Section 6 presents some representative results of system behaviour using real images and the necessity of using the database for a complete identification. Finally, paper conclusions are presented in section 7 .

\section{Image Analysis}

The analysis has been carried out over real aircraft images comprising the tail number, taken from several distances and perspectives. Three types of images have been taken:

- Near images: the tail number is centred in the image, and it is taken orthogonally to the aircraft. Figure 1 shows an example of this type of images.

- Medium distance images, where the tail number appears with other objects (as windows, flags, etc..). Furthermore, the tail number appears distorted by the effect of the aircraft fuselage curvature. Figure 2 shows an example of this type of images.

- Long distance images, where the tail number is confused with the set of objects that appear in the image (wings, motor, soil, sky, staircases, etc..). Figure 3 shows an example of this type of images. 

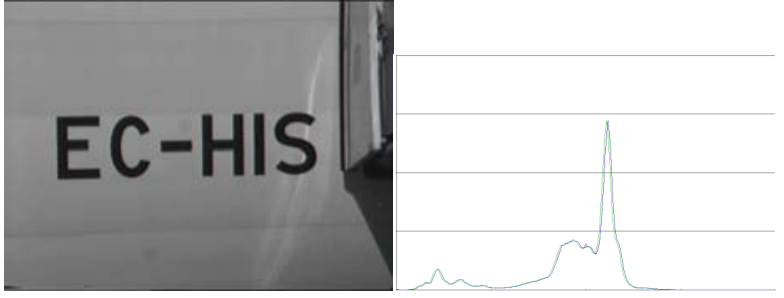

Figure 1. Near image of a tail number and histogram.

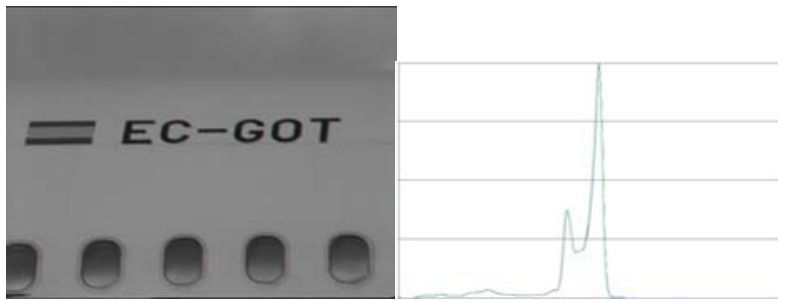

Figure 2. Medium distance image of a tail number and histogram.
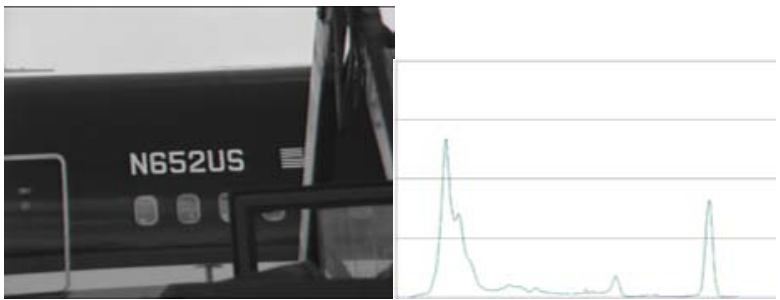

Figure 3. Long distance image of a tail number and histogram.

In addition to the previous considerations, Figures 1, 2 and 3 show that characters can be almost black (Figure 1 and 2 ) or white (Figure 3). An analysis of the histograms of these three example images does not show a peak on characters grey level: they can appear jointly with other zones (windows, flags,..) with similar grey level. The same study considering only the zone surrounding the tail number shows that characters are easy to detect due to its contrast with local background. Grey levels of all characters in a tail number are equal. If we were able to extract the tail number zone, we would have a histogram with two clear peaks: local background and characters. Thus in the Figure 4 we show the histograms for the spatial zones of Figures 1, 2 and 3 where the tail numbers appear.

In addition to the spatial transformations of the characters and the variability of grey level characteristics, Figures 1, 2 and 3 show variations in the letters size. Thus, for example, considering these images, the tail numbers "EC-HIS" and "N652US" sizes are 526 by 134 pixels and 230 by 45 pixels, respectively.
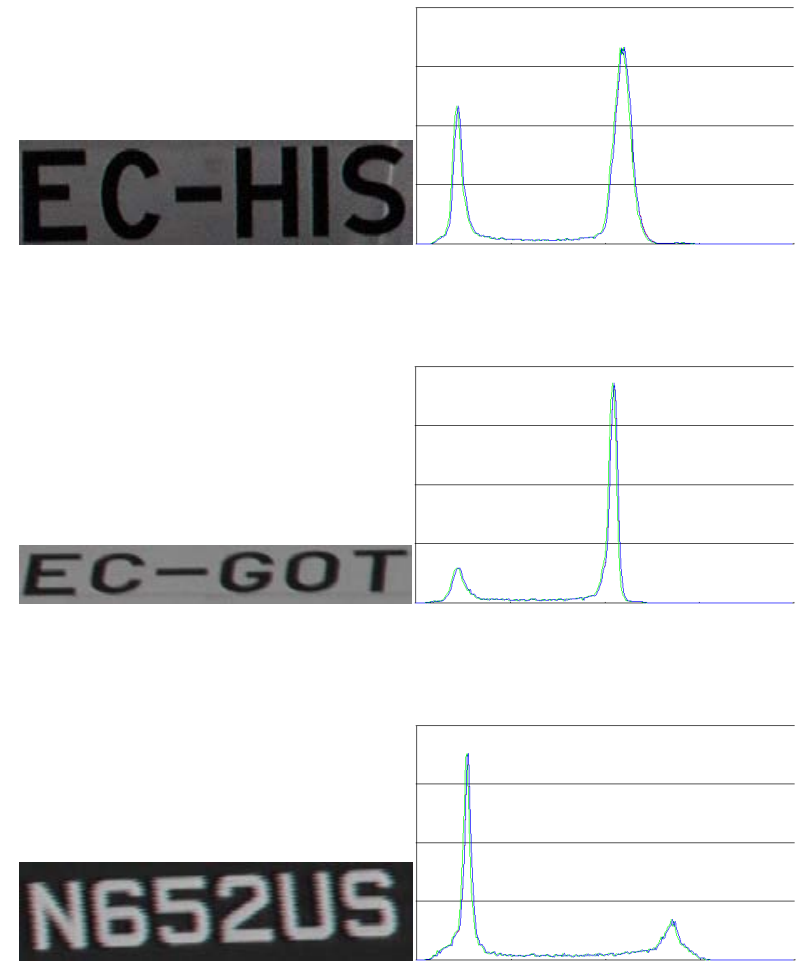

Figure 4. Histograms of Figures 1, 2 and 3 and the spatial zones of the tail number.

From the previous study of tail numbers recorded in the airport and OCR techniques, and studying tail number regulations [12], we have concluded the following points to be used in the aircraft identification algorithm:

- The character and local background grey levels may be different for each tail numbers images. Therefore, the search for feasible regions containing tail numbers should be performed using jointly grey level with spatial information. The whole image and grey level domain (from white to black) should be explored looking for potential tail number regions. All pixels in each tail number have very similar grey levels, quite contrasting with the local background, which is also quite uniform in all images.

- The letter type is similar in all aircrafts but not identical, and substantial differences exist in some letters, specially in the case of numerical characters.

- The analysis of the size of the characters shows a great unpredictable variability in width, height and aspect relationship. Therefore, the use of a correlation techniques is not clear since a standard definition of letters does not exist and, furthermore, the aspect relationship is not maintained due to distortions. The distortions of the characters are not predictable and so it is difficult to adjust the OCR to adapt it to such 
transformations. Meanwhile, the feature-based model defined in previous section is quite robust against these deformations.

- The following aspects of the tail number regulation can be useful:

1. All the characters must have the same height except the character '-' (hyphen).

2. The width of each character is $3 / 4$ of the height. This aspect relation, though, may be corrupted by the projection into camera plane geometry.

3. All the characters should be equally distributed horizontally.

4. All the characters should be aligned horizontally.

\section{All the letters are capital letters.}

6. The hyphen is used to separate the country identification (one or two letters) of the rest of the tail number, but only when the rest starts by a letter character. If the rest of the aircraft starts by a number character, no hyphen is present. For instance, tail numbers as 'N652US' or 'EC-HIS' are correct, while 'N-652US' or 'ECHIS' are not ( $\mathrm{N}$ is the country code for USA and EC the code for Spain).

In Figure 5 there are some additional example figures used in this work. The variability in geometric transformations, intensity and sizes can be observed.
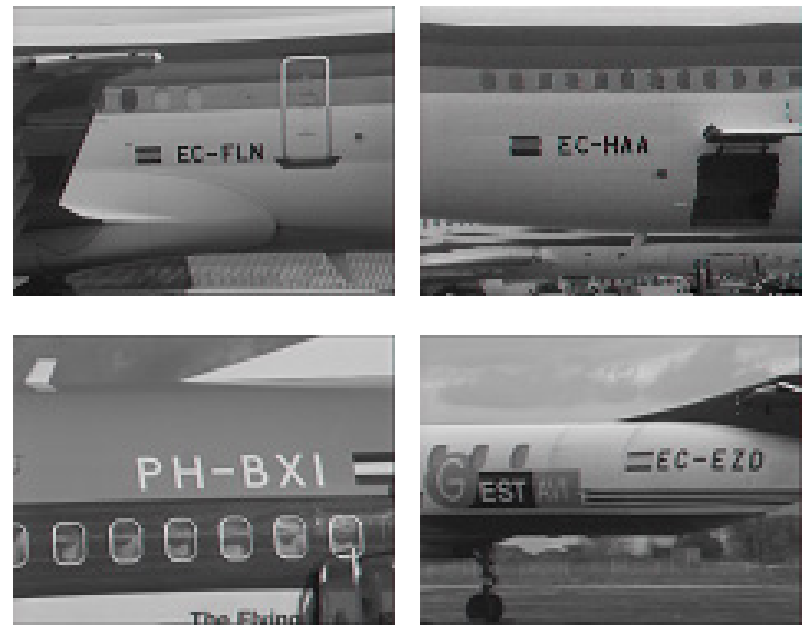
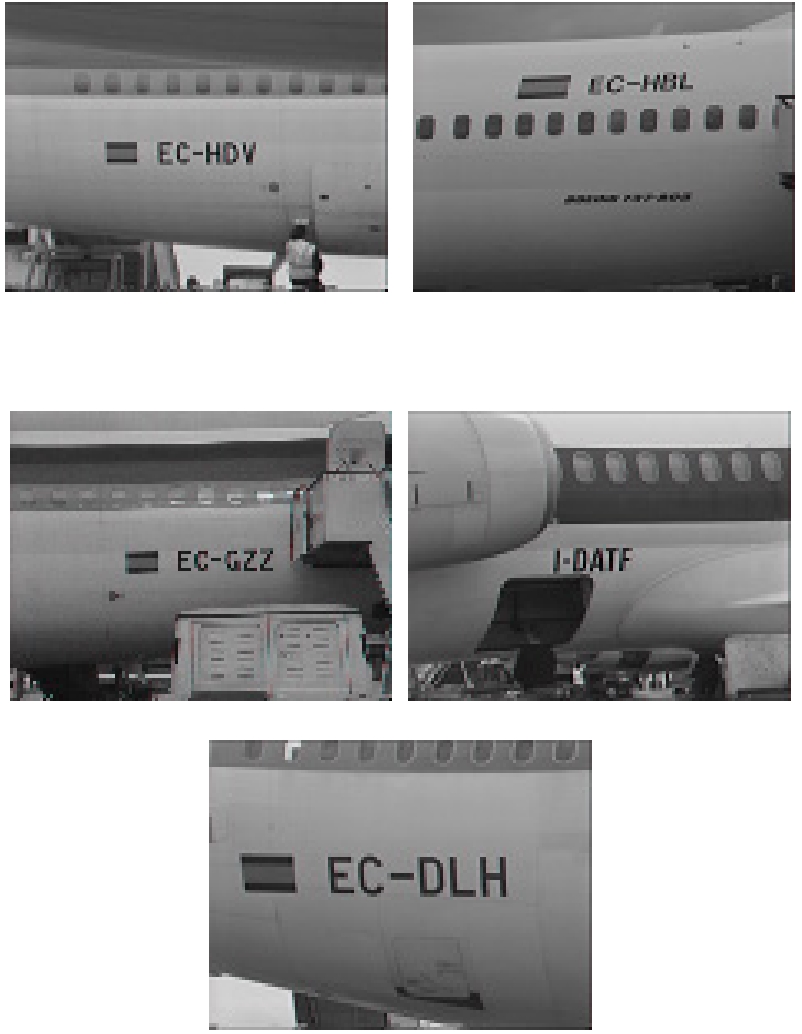

Figure 5. Several test images.

\section{Aircraft Identification System}

Considering these conclusions, the developed system is based basically on three ideas: in the first place, to use the local grey level contrast to detect the characters of the tail number; in second place, to find the zone where the tail number is written to isolate it of the rest of the image; and finally to process this zone to identify the aircraft, using an aircraft database.

The identification system is composed of several subsystems (see Figure 6):

- Process 1 will extract the(s) image(s) when an aircraft must be identified.

- Process 2 will generate a set of sub-images from the same original image proccesing the hystogram of the original image 


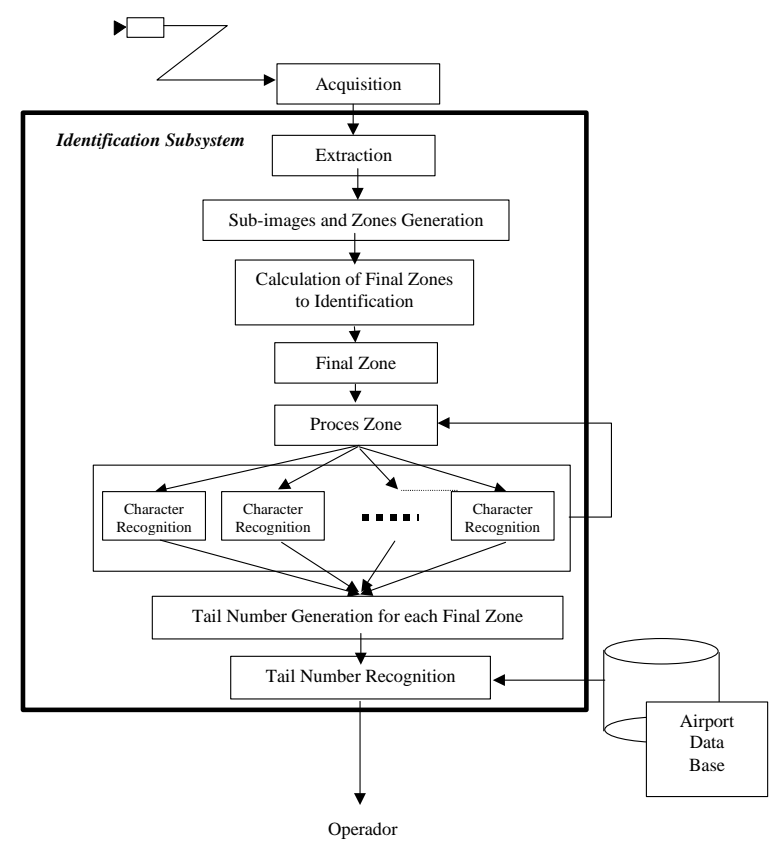

Figure 6. Architecture of the Identification Subsystem.

- Process 3 will process each sub-image, resulting from Process 2 to identify the possible zone in which can be found a tail number. The search process over the grey level is based on the use of two level threshold detectors, whose output is a binary image with a " 1 " in those pixels whose grey level is in the range between both thresholds, and with a " 0 " value if the grey level is outside that range. The procedure is performed iteratively for a set of grey level ranges: each range generates a binary detection image (we will call it a subimage). In a subsequent process we search the tail number location in each sub-image. To do so, we first perform a segmentation over those binary images using a blob growing algorithm [17], finding blobs which may be characters in the tail number, for certain sub-images, or other contrasting features (windows, drawings of the aircraft, ...) for other sub-images. We use the following context information to search for tail numbers: all the characters of the tail number will have the same height and will be found approximately aligned in the same vertical coordinate (all the blobs appear in the same horizontal line), and there are always more than four characters in a tail number. These rules can be used for all the characters, taking into account the exception of the hyphen, which is a special character. It does not have the same height as the rest of the letters, nor it is vertically aligned, but it may be easily identified since it is located near the vertical centre of the tail number region. Applying these rules a set of candidate tail number regions can be defined for each sub-image.
- Process 4 will limit the number of possible final zones through the fusion and the elimination of previous zones. The result of process 3 will be a set of potential tail number zones, several for each sub-image. We have defined a procedure to limit the number of potential candidate regions to be analysed searching tail number. The set of two thresholds detectors used for the calculation of the sub-images are defined with overlapping ranges, so that the blobs of the characters may be detected in several sub-images. Using this fact, we may fuse the candidate regions from several subimages making some validation tests over the spatial position of the candidate regions: each candidate region must appear in several sub-images and they should be almost equal for several subimages After this, the systems will have only a few candidate regions. Typically, they are the region containing the tail number, another region containing the aircraft windows (if they appear in the image), and sometimes other texts written in the fuselage, such as the aircraft manufacturer or model.

- Process 5 will process of each final zone to eliminate the noise and other effects that they could appear. From now, each candidate region will be treated as an independent image. This image will be processed to increase the character definition before attempting to identify the character. First of all, a two-level threshold detector similar to the preceding ones, but with its thresholds calculated from those used to define the subimages fused to form this candidate, is used to detect the final blobs to be analysed by the identification system. Then, some slight morphological processing is performed, in order to be able to use an additional feature for the aircraft recognition, which is the number of holes in the character [19]. The threshold detector sometimes suffer some problems leading to tiny holes in the blob, which would corrupt the character recognition procedures. This morphological processing is only used to overcome this problem, and it must not change too much blobs shape.

- Process 6 focus the attention on the final zone to isolate each character that compose it.

- Process 7 evaluates the probability for each character to be a predefined pattern using the distance between the pattern definition and the tentative character (equation 1). 


$$
p\left(C, P_{i}\right)=\frac{e^{--\frac{d\left(C, P_{i}\right)}{D}}}{\sum_{j=1}^{35} e^{-\frac{d\left(C, P_{j}\right)}{D}}}
$$

where $\mathrm{C}$ is the extracted character, $\mathrm{P}_{\mathrm{i}}$ is the pattern (there are 35 patterns : capital letters, number and the hyphen), $\mathrm{d}($.$) is the distance$ between extracted character and the pattern and it is explained in section 4 .

- Process 8 evaluates the joint probability distribution using the previous value of probability and the airport database. The identification method starts requesting all those tail numbers. Let us first imaging all tail numbers in the database had the same length $(\mathrm{N})$ and there were only one candidate zone (the correct one) comprising a number of tentative characters equal to that length. In that case, the method to be used would be just searching for the maximum joint probability, calculated for each tail number in the database as:

$$
P_{\text {tail-number }}=\prod_{i=1}^{N} p\left(C_{i}, P_{\text {tail-number }(i)}\right)
$$

where $\mathrm{P}_{\text {tail-number(i) }}$ is the pattern associated to the i-th character in the tail number, $\mathrm{C}_{\mathrm{i}}$ is the tentative character at the $\mathrm{i}$-th position in the candidate region, and $\mathrm{p}\left(\mathrm{C}, \mathrm{P}_{\mathrm{i}}\right)$ are the previous probability.

\section{Distance and character probability evaluation}

The character identification procedure is based on an expert system calculating a "probability" that the tentative character correspond to each one of the potential patterns. This expert system uses, first of all, the global density of the character to classify directly a pair of special characters: the "I" and the hyphen. The global density of a character is defined as the number of character pixels divided by the pixels of the rectangular zone circumscribing the character. In this sense, the hyphen and the character "I" has an ideal density value of $100 \%$. The rest of characters has a value lower than $90 \%$. This information is used by expert system to classify the hyphen, the character "I" and to mark spurious characters (for example, flags or windows the preprocessing considers as tentative characters). Besides, the expert system uses the height and width of the tentative character to detect the hyphen or the character "I". The rest of the characters are evaluated using the difference in the number of holes and a weighted Euclidean distance between the vectors (comprising the nine per-sector densities) of tentative characters and predefined patterns.
An example of the calculation of the Euclidean distance is described in figure 7 .
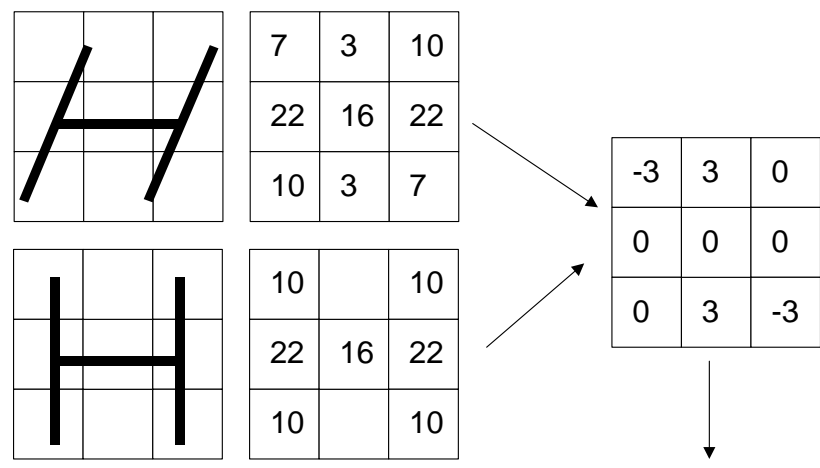

$$
\sqrt{\sum_{i=1}^{9} \alpha_{i}\left(s_{i}^{\text {tentative }_{i}}-s_{i}^{\text {pattern }}\right)}
$$

Figure 7: Vector definition of character $\mathrm{H}$.

The proposed procedure measures the distance between the recognized character and the ideal patterns of the characters set $\left(d\left(C, P_{i}\right)\right)$ and then, a character identification probability is calculated for each pattern. This "probability" value reflects the matching of each a priory pattern to the tentative character obtained from the image, and is calculated using equation (1).

D is a constant parameter (we will call it "characters distance dispersion"), being its value critical to the discrimination performance of the OCR, and the summation in the denominator is performed over all potential patterns. Higher D values lead to a non too discriminating classifier, while lower D may introduce erroneous classifications of one letter, too difficult to recover by the joint probability maximization procedure. Several distances definitions (namely, those deriving from 1-norm, 2-norm and $\infty$-norm) were evaluated and the Euclidean distance (deriving from 2-norm) obtained the best performance.

We noted the identification system performance could be refined using weights to prioritize some parts of the characters. It is reasonably supposed that the relevance of every sector is different in order to discriminate different patterns. For example, the density of central sector discriminates quite well. The patterns with a central hole ("O", "D", "Q", "G", "U") are clearly distinguished of the rest. On the contrary, the first sector does not incorporate much information as all patterns have similar density values. Thus the distance measure may be modified to incorporate this domain information and a global optimization technique to find the sectors weight is 
required. The weighted Euclidean distance is defined in equation (3).

$$
\begin{aligned}
& d\left(C, P_{i}\right)=\sqrt{\sum_{j=1}^{9} \alpha_{j}\left(c_{j}-s_{j}\right)^{2}} \\
& \sum_{i=1}^{9} \alpha_{i}=9
\end{aligned}
$$

In this identification model the optimal (in terms of overall system performance) $D$ and $\alpha_{j}$-values are correlated. Therefore, we applied a global optimisation procedure to simultaneously adjust all of them automatically [20]. An evolutionary strategy has been applied to obtain these values [21]. Thirty optimisation processes (the number of runs necessary has been calculated using the method suggested by Schwefel [22]) have been performed.

\section{Aircraft identification method}

The method used to identify an aircraft is based on the use of a database, in which tail numbers for every aircraft in the airport are included. The identification method starts requesting all those tail numbers. Let us first imaging all tail numbers in the database had the same length $(\mathrm{N})$ and there were only one candidate zone (the correct one) comprising a number of tentative characters equal to that length. In that case, the method to be used would be just searching for the maximum joint probability, calculated for each tail number in the database using equation (2).

The problem is there are tail numbers with different lengths (we will call this length $\mathrm{N}_{\text {tail_number }}$ ) and sometimes spurious tentative characters may appear both at the beginning and end of the candidate regions, so that the number of characters in the region $\left(\mathrm{N}_{\text {region }}\right)$ is different. To overcome this problem, for each tail number, an sliding window with a length equal to the number of characters has been used to select tentative characters from the image. This way, we would have $\mathrm{N}_{\text {region }}-\mathrm{N}_{\text {tail_number }}+1$ hypothetic tentative character arrays of length $\mathrm{N}_{\text {tail_number }}$ for this tail number. We should select, among all those arrays, the one providing a higher probability according to (2). If $\mathrm{N}_{\text {tail_number }}$ is higher than $\mathrm{N}_{\text {region}}$, the tail number is discarded as a potential candidate. Finally, all candidate regions from the tail-number search algorithm should be explored, selecting the one providing the highest probability.

\section{Experiments with airport images}

We have tested the behaviour of the described system with 105 real images taken in Barajas Airport. They were recorded from 60 different tail numbers, viewed from different positions. All tail numbers were correctly identified using a database in which the 60 aircraft tail numbers were included.

To see how robust our system is we have defined a quality statistic, which is the posterior probability of the identified tail number, assuming that all tail numbers in the database have equal prior probability. This posterior probability could be calculated as:

$$
P_{\text {posterior }}=\frac{\max \left(P_{\text {tail_number }}\right)}{\sum_{\text {all tail numbers }} P_{\text {tail_number }}}
$$

This probability indicates the confidence we have in our identification. We only have problems with four images in our data set. They appear for some cases with quite similar tail numbers. Concretely, the problems appear for two tail numbers, with images taken near and far away. They are EC-HDM and, specially, EC-HGA. The problematic situation appears because there are several competing tail numbers with only one letter of difference. For the EC-HDM case, in the database we also have ECHDL, EC-HDU and EC-HDV. And for the EC-HGA tail number, we have EC-HAA, EC-HGO, EC-HGU and ECHGX. It should be noted this posterior probability could be used to assess the confidence we have in our detection. And in not too clear cases, a most complex algorithm could be used to ensure the correct decisions.

\section{Conclusions}

An identification subsystem has been developed to be integrated in a video surveillance system. The identification algorithm has been developed taking into account the wide variability of aircraft real images taken in Madrid/Barajas airport. The proposed algorithm has been shown to be robust in its application.

The identification procedure extracts the tail number region from a image. This region is processed to enhance the contrast of characters. Then an OCR algorithm based on feature representation of characters is applied. In order to incorporate some heuristics (taken from ICAO normative) an expert system has been developed. This expert system evaluates for each tentative character (extracted from the image) the probability to be a predefined pattern. Using the set of tail numbers stored in the airport database, a joint probability distribution is evaluated for each of them, and the tail number with the highest value is identified.

The reliability and precision of the system has been determined using a set of 105 real images. The 
identification system obtains a correct identification of all those aircraft using the airport database.

\section{Acknowledgments}

The authors recognize the support provided by AENA (Aeropuertos Españoles y Navegación Aérea) with special thanks to Angeles Varona and Germán Gonzalez for their help during the work of obtaining the database of aircraft images.

\section{References}

[1] "European Manual of Advanced Surface Movement and Control Systems (ASMGCS)". Draft. Volume 1: Operational Requirements. Draft Version 04. 08/24/2000.

[2] Proceedings of the ECAC APATSI and EC Workshop on A-SMGCS. Frankfurt, Germany. April 1994.

[3] "Manual of SMGCS. ICAO". Doc. 9476-AN/927

[4]. Schwabn C. E, Rost D. P.. "Airport Surface Detection Equipment" Proceedings of the IEEE, No. 2, February 1985.

[5] "Rapport d'evaluation du système SYLETRACK". ADP. March 1997.

[6] "Cooperative Area Precision Tracking System (CAPTS). Final report of test results". Frankfurt Airport. Airsys ATM Gmbh. January 1998.

[7] Lipton A.J., Fujiyoshi H., Patil R.S., "Moving Target Classification and Tracking from Real-Time Video", Proc. IEEE Image Understanding Workshop, pp. 129-136, 1998.

[8] Beymer D., McLauchlan P., Coifman B., Malik J., "A real-Time Computer Vision System for Measuring Traffic Parameters", Proc. Computer Vision and Pattern Recognition, June 1997.

[9] Special Section on Video Surveillance, IEEE Trans on PAMI, vol. 22, no. 8, Aug. 2000.

[10] Besada J., García J., Varona A., González G., Molina J. M., Portillo J.. "Image-Based Automatic Surveillance For Airport Surface", 4th International Conference on Information Fusion, Fusion 2001, pp (WeA1) 11-18. Montreal, Canadá, Agosto 2001.
[11] Jain R., Kasturi R., R.G., B.G. Schunck, "Machine Vision", McGraww-Hill, 1995.

[12] ICAO, Convention on Civil Aviation, 1981.

[13] Sanka M, Hlavac V., Boyle R., "Image Processing, Analysis and Machine Vision", Brooks/Cole Publishing Company, 1999.

[14] Castleman K. R.. "Digital Image Processing". Prentice Hall. 1996.

[15]. J.C Russ, "The Image Processing Handbook", CRC Press, 1995.

[16] Pratt W. K., "Digital Image Processing", John Wiley \& Sons, 1991.

[17] Parker J.R., "Algorithms for Image Processing and Computer Vision”, Willey 1996.

[18] Mori S., Suen C. Y., Yamamoto K., "Historical Review of OCR Research and Development ", Proceedings of the IEEE, Vol. 80, No. 7, pp. 1029-1058, July 1992.

[19] Giardina C.R., Dougherty E.R., "Morphological Methods in Image and Signal Processing", Prentice-Hall, 1988.

[20] Törn A., Zilinskas A., "Global Optimization”, vol. 350 of Lecture Notes in Computer Science. Springer, Berlin. 1991.

[21] Bäck T. "Evolutionary Algorithms in Theory and Practice", Oxford University Press, 1996.

[22] Schewefel H.P. "Evolutionary learning optimumseeking on parallel computer architectures". In A. Sydow, S. G. Tzafestas, and R. Vichnevetsky, editors, Proceedings of the International Symposium on Systems Analysis and Simulation 1988, I: Theory and Foundations, pp. 217-225. Akademie-Verlag, Berlin. 1988. 